\title{
The Mechanisms Responsible for Lack of Reproducible Induction of Atrioventricular Nodal Reentrant Tachycardia
}

\author{
S. ADAM STRICKBERGER, M.D., EMILE G. DAOUD, M.D., \\ MARK J. NIEBAUER, M.D., CAROL HASSE, R.N., K. CHING MAN, D.O., \\ and FRED MORADY, M.D.
}

From the Division of Cardiology, Department of Internal Medicine, University of Michigan Medical Center, Ann Arbor, Michigan

Lack of AVNRT Induction. Introduction: AV nodal reentrant tachycardia (AVNRT) is not always reproducibly inducible. The purpose of this study was to determine the mechanisms responsible for the lack of reproducible induction of AVNRT.

Methods and Results: The induction of AVNRT was assessed with atrial burst pacing, and with atrial and ventricular programmed stimulation, each with one and two extrastimuli, in 103 patients with AVNRT. The stimulation protocol was repeated 10 times in the baseline state, during isoproterenol infusion, and after atropine administration, or until AVNRT was induced in 7 of 10 attempts. The mechanisms responsible for $<7$ of 10 inductions were classified as: (1) the inability to achieve critical AH prolongation; (2) fast pathway block; and (3) slow pathway block. The induction endpoint was achieved in 90 patients: 55 in the baseline state, 34 during isoproterenol infusion, and 1 after atropine. The mechanism of noninducibility in the baseline state $(n=48)$ was the inability to achieve a critical AH interval in $20 \%$, fast pathway block in $49 \%$, and slow pathway block in $31 \%(\mathrm{P}=0.02)$. During isoproterenol administration $(n=14)$ and after atropine administration $(n=13)$, the three mechanisms were equally responsible for nonreproducible induction of AVNRT.

Conclusions: The induction of AVNRT is poorly reproducible in approximately $10 \%$ of patients. In the baseline state, the most common reason for the inability to reproducibly induce AVNRT is fast pathway block. In the presence of isoproterenol or atropine, each of the three mechanisms was equally responsible for noninducibility of AVNRT. ( $J$ Cardiovasc Electrophysiol, Vol. 7, pp. 494-502, June 1996)

tachycardia induction, reentry, arrhythmia mechanism

\section{Introduction}

Although AV nodal reentrant tachycardia (AVNRT) is usually inducible by atrial or ventricular pacing, sometimes it may not be reproducibly inducible or may require isoproterenol or atropine administration for induction. ${ }^{1,2}$ No prior study has quantitated the mechanisms responsi-

Address for correspondence: S. Adam Strickberger, M.D., Division of Cardiology, Department of Internal Medicine, University of Michigan Medical Center, 1500 E. Medical Center Drive, Box 0022, Ann Arbor, MI 48109. Fax: 313-936-7026.

Manuscript received 15 November 1995; Accepted for publication 5 March 1996. ble for the nonreproducible induction of this arrhythmia. Therefore, the purpose of this study was to determine the mechanism(s) responsible for lack of reproducible induction of AVNRT.

\section{Methods}

\section{Patient Characteristics}

The subjects of this study were 103 consecutive patients ( 24 male and 79 female; mean age $55 \pm 7$ years) with paroxysmal supraventricular tachycardia (PSVT) who underwent an electrophysiologic test and were found to have typical AVNRT. None of the patients had structural heart 
disease. The patients had been symptomatic from AVNRT for $16 \pm 12$ months.

\section{Electrophysiologic Testing}

Electrophysiologic tests were performed in the postabsorptive state after informed consent was obtained and after all antiarrhythmic medications had been discontinued for at least five half-lives. Three 7-French quadripolar electrodes were inserted in a femoral vein and positioned in the high right atrium, across the tricuspid valve to record the His-bundle electrogram, and in the right ventricle. Leads $\mathrm{V}_{1}, \mathrm{I}, \mathrm{II}$, and III and the intracardiac electrograms were displayed on an oscilloscope and recorded on a Mingograf 7 recorder (SiemensElema, Solna, Sweden). Pacing was performed with a programmable simulator (Bloom Associates, Reading, PA, USA). The electrophysiologic test was directed at inducing and determining the mechanism of PSVT, as well as measuring the conduction properties and the refractory periods of the AV node. ${ }^{1}$ If the PSVT could not be provoked in the baseline state, programmed stimulation was repeated during infusion of $2 \mu \mathrm{g} / \mathrm{min}$ of isoproterenol. Incremental pacing and programmed simulation were performed from the right atrium and right ventricle to define anterograde and retrograde $\mathrm{AV}$ nodal conduction properties and refractoriness, and to confirm that the provoked arrhythmia was in fact AVNRT. Typical AVNRT was diagnosed using previously established criteria. ${ }^{3}$

\section{AVNRT Induction Protocol}

After the tachycardia was induced once and the diagnosis of typical AVNRT was confirmed, patients underwent the investigational protocol, which was approved by the University of Michigan Committee on Human Research. The stimulation protocol consisted of 18 steps (Table 1). Patients only underwent the subsequent step of the induction protocol if the previous one did not achieve the endpoint of sustained AVNRT induction in at least 7 of 10 attempts. AVNRT that persisted for at least 30 seconds was defined as sustained. The first step of the stimulation protocol used incremental atrial pacing starting at $30 \mathrm{msec}$ longer than the AV block cycle length. One attempt was defined as constant atrial pacing at $30 \mathrm{msec}$ above the AV block cycle length, then $20 \mathrm{msec}$ above the AV block cycle length, $10 \mathrm{msec}$ above the AV block cycle length, at the AV block cycle length, and finally at $10 \mathrm{msec}$ below the AV block cycle
TABLE 1

18-Step Pacing Protocol

\begin{tabular}{|c|c|c|}
\hline Step No. & $\begin{array}{c}\text { Pharmacologic } \\
\text { State }\end{array}$ & Pacing Protocol \\
\hline 1 & B & $\begin{array}{l}\text { Incremental atrial overdrive } \\
\text { pacing }\end{array}$ \\
\hline 2 & B & $\begin{array}{l}\text { Atrial programmed } \\
\text { stimulation with } \mathrm{A} 2\end{array}$ \\
\hline 3 & B & $\begin{array}{l}\text { Atrial programmed } \\
\text { stimulation with } \mathrm{A} 2 \mathrm{~A} 3\end{array}$ \\
\hline 4 & B & $\begin{array}{l}\text { Incremental ventricular } \\
\text { overdrive pacing }\end{array}$ \\
\hline 5 & B & $\begin{array}{l}\text { Ventricular programmed } \\
\text { stimulation with V2 }\end{array}$ \\
\hline 6 & B & $\begin{array}{l}\text { Ventricular programmed } \\
\text { stimulation with } \mathrm{V} 2 \mathrm{~V} 3\end{array}$ \\
\hline 7 & I & $\begin{array}{l}\text { Incremental atrial overdrive } \\
\text { pacing }\end{array}$ \\
\hline 8 & I & $\begin{array}{l}\text { Atrial programmed } \\
\text { stimulation with } \mathrm{A} 2\end{array}$ \\
\hline 9 & I & $\begin{array}{l}\text { Atrial programmed } \\
\text { stimulation with } \mathrm{A} 2 \mathrm{~A} 3\end{array}$ \\
\hline 10 & I & $\begin{array}{l}\text { Incremental ventricular } \\
\text { overdrive pacing }\end{array}$ \\
\hline 11 & I & $\begin{array}{l}\text { Ventricular programmed } \\
\text { stimulation with V2 }\end{array}$ \\
\hline 12 & I & $\begin{array}{l}\text { Ventricular programmed } \\
\text { stimulation with } \mathrm{V} 2 \mathrm{~V} 3\end{array}$ \\
\hline 13 & A & $\begin{array}{l}\text { Incremental atrial overdrive } \\
\text { pacing }\end{array}$ \\
\hline 14 & A & $\begin{array}{l}\text { Atrial programmed } \\
\text { stimulation with A2 }\end{array}$ \\
\hline 15 & A & $\begin{array}{l}\text { Atrial programmed } \\
\text { stimulation with } \mathrm{A} 2 \mathrm{~A} 3\end{array}$ \\
\hline 16 & A & $\begin{array}{l}\text { Incremental ventricular } \\
\text { overdrive pacing }\end{array}$ \\
\hline 17 & A & $\begin{array}{l}\text { Ventricular programmed } \\
\text { stimulation with } \mathrm{V} 2\end{array}$ \\
\hline 18 & A & $\begin{array}{l}\text { Ventricular programmed } \\
\text { stimulation with } V_{2} V_{3}\end{array}$ \\
\hline
\end{tabular}

$\mathrm{A}=$ atropine; $\mathrm{B}=$ baseline; $\mathrm{I}=$ isoproterenol; $\mathrm{A} 2=\sin -$ gle atrial extrastimulus; $\mathrm{A} 2 \mathrm{~A} 3$ = double atrial extrastimuli; $\mathrm{V} 2=$ single ventricular extrastimulus; $\mathrm{V} 2 \mathrm{~V} 3=$ double ventricular extrastimuli.

See text for complete description of pacing protocol.

length. During atrial pacing, the S1s were delivered until $\mathrm{AH}$ prolongation and subsequent $\mathrm{AH}$ block occurred or, if $\mathrm{AH}$ block did not occur, for at least 5 seconds. As with each step of the protocol, this was repeated 10 times. If the AVNRT induction endpoint was not reached, the second step of the protocol, programmed stimulation of the atria (A1) with a single premature atrial extrastimulus (A2) was performed. A1A2 stimulation was performed with eight S1s and with at least two basic drive cycle lengths $(600,500,400,350$ $\mathrm{msec})$. The A1A2 coupling interval was decremented in 10-msec steps until AVNRT was induced or until the atrial or AV nodal refractory period was reached. The third step consisted of programmed atrial stimulation with two extrastimuli (A2A3). The third atrial extrastimulus was decre- 
mented in a manner similar to that described for A2. The A1A2 coupling interval was selected to be 10 to $30 \mathrm{msec}$ longer than the fast pathway effective refractory period. The fourth step used fixed rate right ventricular pacing at 30,20 , and $10 \mathrm{msec}$ above the VA block cycle length, at the VA block cycle length, and at $10 \mathrm{msec}$ shorter than the VA block cycle length. One attempt was defined in a manner similar to that described above in step 1 for fixed rate atrial pacing. Step 5 was programmed stimulation of the right ventricle with one extrastimulus, and step 6 was with two extrastimuli. When two ventricular extrastimuli were used, the V1V2 coupling interval was the shortest coupling interval associated with VA conduction. When the endpoint of at least 7 of 10 successful inductions of AVNRT was not achieved in the baseline state with steps 1 through 6 , each step was repeated during the infusion of $2 \mu \mathrm{g} / \mathrm{min}$ of intravenous isoproterenol infusion (steps 7 to 12). If the endpoint was not achieved during isoproterenol administration, the infusion was discontinued. After at least 20 minutes, atropine $(1 \mathrm{mg})$ was injected, and pacing steps 13 to 18 were performed (Table 1).

In accord with the selection criteria for this study, all patients had AVNRT that could be provoked at least once, allowing the diagnosis to be established. However, in some patients, after the diagnosis of AVNRT was established, the arrhythmia could not be induced again despite performing steps 1 through 18 .

\section{Mechanisms of Noninduction of AVNRT}

The general mechanisms responsible for noninducibility of a reentrant tachycardia include the inability to initiate reentry and the inability to maintain reentry. More specifically, when AVNRT could not be reproducibly provoked, 1 of 3 mechanisms for noninduction was identified. The first mechanism, an example of the inability to initiate reentry, was the inability to attain the critical atrialHis $(\mathrm{AH})$ interval required for induction of AVNRT. This mechanism was determined to be responsible if the maximum $\mathrm{AH}$ during failed attempts to induce AVNRT was shorter than the shortest AH present when tachycardia was induced (Fig. 1). The second possible mechanism was block in the fast pathway. When manifest as a prolonged VA block cycle length (> $350 \mathrm{msec}$ ), absent VA conduction, ${ }^{4}$ or critical $\mathrm{AH}$ prolongation without an AV nodal echo, this would be another example of failure to initiate reentry. When a few echoes occurred and termination was due to retrograde fast pathway block, this was an example of the inability to maintain reentry (Fig. 2). The third possible mechanism was block in the slow pathway. An AV block cycle length $>400$ msec would prevent tachycardia induction, ${ }^{4}$ while a retrograde $\mathrm{AV}$ nodal echo(s) with subsequent anterograde slow pathway block would prevent maintainance of the tachycardia (Fig. 3).

\section{Statistical Analysis}

Continuous variables are expressed as mean \pm $1 \mathrm{SD}$ and were compared using a $t$-test for paired or unpaired variables. Chi-square analysis was used to compare the frequencies of the mechanisms responsible for inability to AVNRT. P values < 0.05 were considered statistically significant.

\section{Results}

\section{Inducibility}

The endpoint of AVNRT induction in at least 7 of 10 attempts was achieved in 90 of the 103 patients (Fig. 4). The number of AVNRT inductions in the remaining 13 patients ranged from 0 to 6 (Fig. 4).

\section{Mode of AVNRT Induction}

Following the initial induction required to establish the diagnosis of the tachycardia, only steps $1,2,4,7,8$, and 13 of the pacing protocol achieved the endpoint of inducible AVNRT in at least 7 of 10 attempts (Fig. 5). This included 84 patients who were inducible with fixed rate atrial pacing (steps 1,7 , and 13), 5 who were induced with atrial programmed stimulation with a single atrial extrastimulus (steps 2 and 8), and 1 patient in whom burst ventricular pacing (step 4) was effective. Atrial programmed stimulation with two atrial extrastimuli (steps 3, 9, and 15) and ventricular programmed stimulation with one and two extrastimuli (steps 5, 6, 11, 12, 17, and 18) did not result in achievement of the endpoint in any patient.

\section{Drug State During AVNRT Induction}

Among the 90 patients in whom AVNRT was induced in at least 7 of 10 attempts, after the initial induction required to establish the diagnosis of the tachycardia, 55 had the endpoint reached in the 

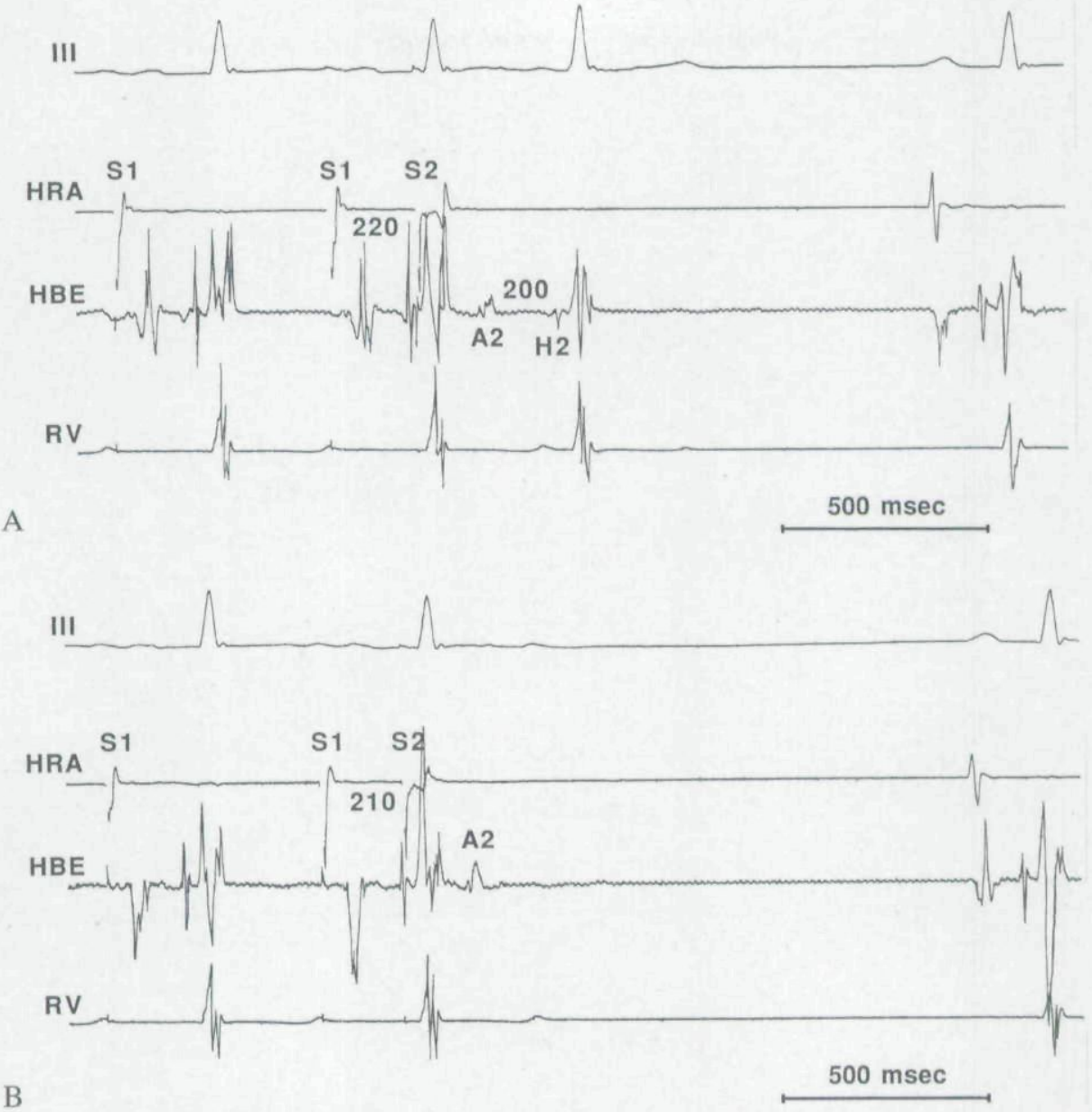

Figure 1. Inadequate AH prolongation preventing AVNRT induction. (A) The shortest AlA2 (310 msec) that conducts through the AV node ( $A 2 \mathrm{H} 2200 \mathrm{msec}$ ) is shown. (B) The subsequent AlA2 (300 msec) blocks in the AV node, demonstrating the effective refractory period of the AV node. The critical AH for induction of AVNRT was $290 \mathrm{msec}$ in this patient. The drive train stimuli (S1) and the atrial extrastimulus (S2) are shown. The atrial depolarization that results from the S2 is labeled A2. The subsequent His-bundle depolarization is labeled H2. The figure is organized from top to bottom with lead III, the high right atrial bipolar electrogram recording (HRA), the His-bundle bipolar electrogram recording (HBE), and the right ventricular bipolar electrogram recording $(R V)$.

baseline state, 34 during isoproterenol infusion, and 1 after the administration of atropine. One patient who did not have inducible AVNRT in the baseline state or during isoproterenol infusion did not receive atropine because of a history of atropine intolerance. There was no difference in identifiable baseline clinical characteristics of patients who attained the AVNRT induction endpoint in the baseline state versus during drug administration. However, there were several electrophysiologic characteristics that were different between patients who achieved the induction endpoint in the baseline state and those who did not (Table 2). The electrophysiologic parameters of patients who required isoproterenol to achieve the induction endpoint are shown in Table 3.

\section{Mechanisms Responsible for Noninducibility of AVNRT}

After the initial induction of AVNRT to establish the diagnosis, the endpoint of 7 or more AVNRT inductions out of 10 attempts was not achieved in 48 patients in the baseline state (Table 4). Among these 48 patients, 14 did not achieve the endpoint during isoproterenol administration and 12 did not achieve the endpoint after atropine administration. Therefore, there were a total of 74 induction attempts that did not attain the endpoint of at least seven inductions of AVNRT. The mechanisms responsible for not achieving the endpoint were the inability to attain a critical $\mathrm{AH}$ interval in 22 patients $(30 \%)$, failure of conduc- 

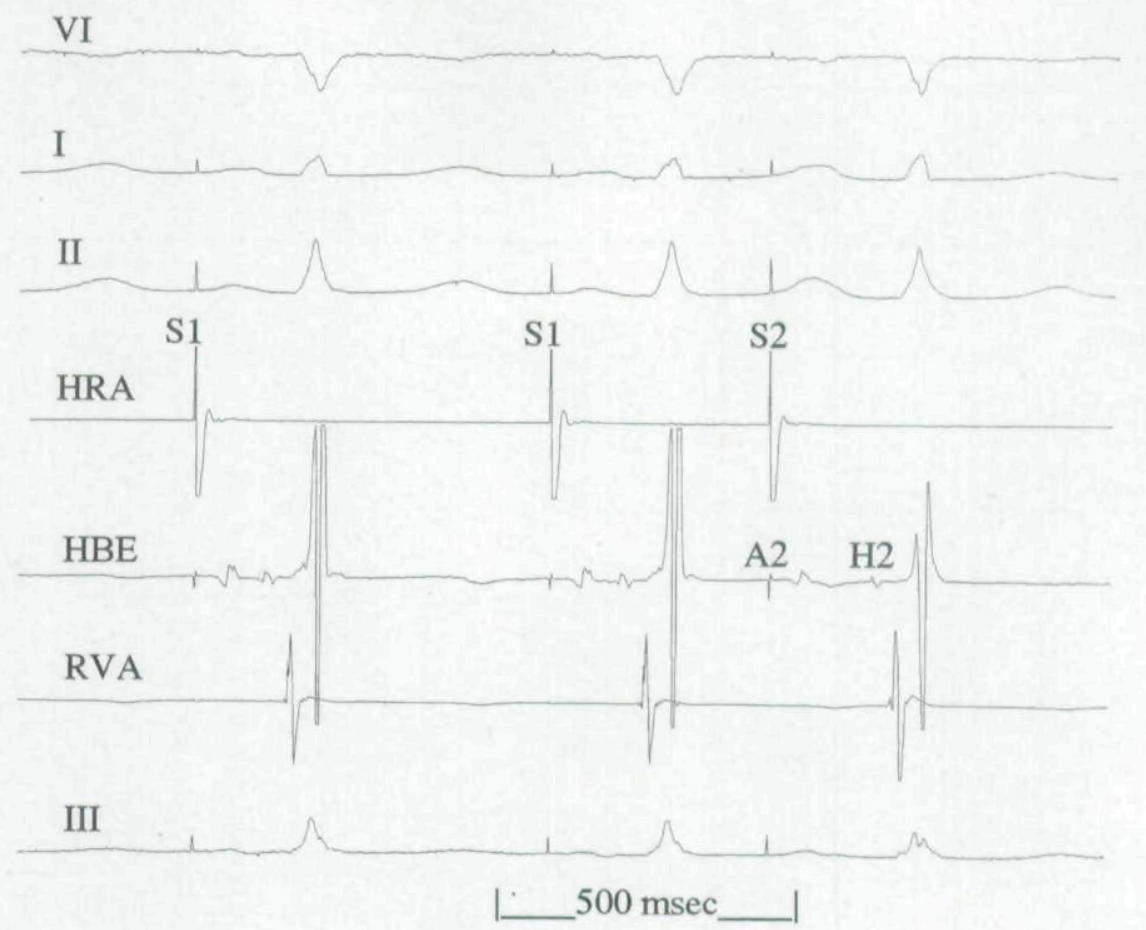

A
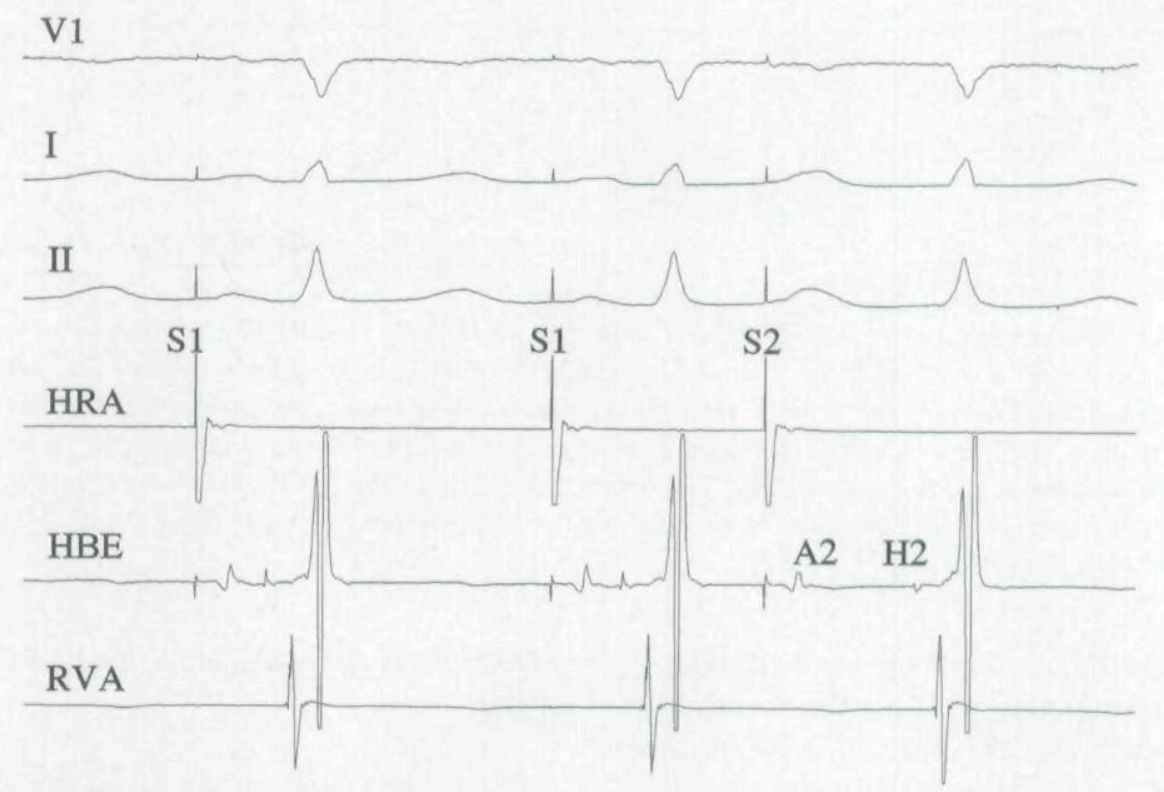

III

B

$500 \mathrm{msec}$

Figure 2. These recordings demonstrate fast pathway conduction block limiting the induction of AVNRT. (A) Anterograde fast pathway conduction with an A1A2 of $370 \mathrm{msec}$ and an A2H2 of $130 \mathrm{msec}$ is shown. (B) An AlA2 of 360 msec is associated with fast pathway conduction block and anterograde slow pathway conduction ( $A 2 H 2240 \mathrm{msec})$. Despite the jump, the tachycardia is not induced due to retrograde fast pathway block. 

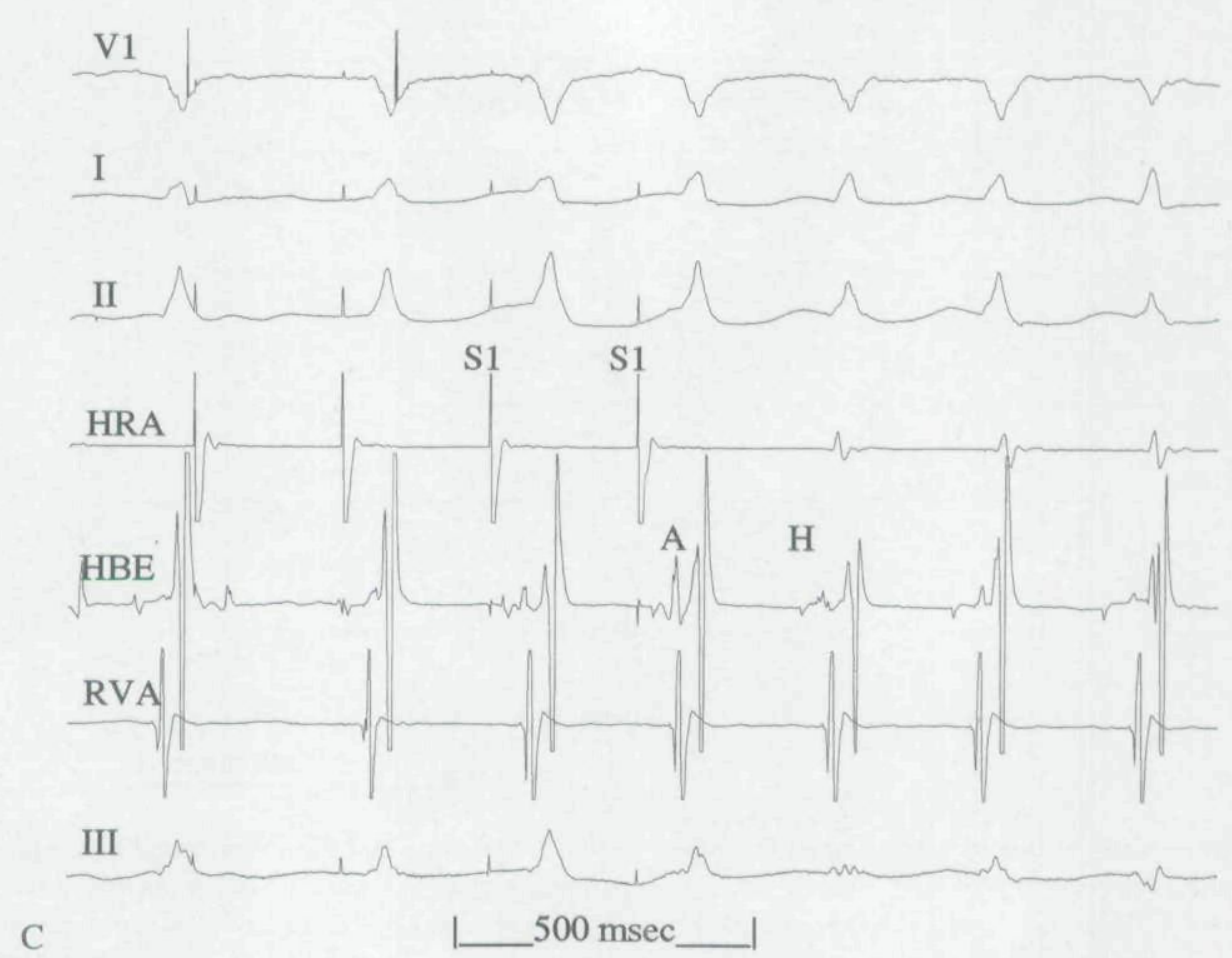

Figure 2. (Continued) (C) Critical prolongation of the AH interval is demonstrated. With fixed rate atrial pacing at $250 \mathrm{msec}$, critical AH prolongation occurs $(230 \mathrm{msec})$, the retrograde fast pathway is no longer refractory, and the tachycardia is initiated. The tracings and abbreviations are as described in Figure 1, except lead II is also shown.

tion in the fast pathway in 27 patients (36\%), and failure of slow pathway conduction in the remaining 25 patients (34\%). There was one patient in whom two mechanisms were identified as the etiology of noninduction in the baseline state. Both of these mechanisms were included in the analysis.

In the baseline state, there were 48 patients in whom AVNRT was inducible in $<7$ of 10 attempts. The inability to achieve a critical $\mathrm{AH}$ interval was the mechanism responsible in $20 \%$, fast pathway conduction block was responsible in $49 \%$, and slow pathway block was the mechanism responsible in $31 \%$ of the patients $(P=0.02)$. The same mechanism was responsible for noninduction in each step of the protocol, except in one patient in whom the lack of induction during step 1 was due to the inability to achieve a critical $\mathrm{AH}$ interval, while the mechanism in the remaining steps during the baseline state was slow pathway block. There was only one patient in whom step 4 (fixed rate ventricular pacing) resulted in achievement of the endpoint. In this patient, during atrial pacing (steps 1 through 3), AVNRT induction was limited by retrograde fast pathway block.

During isoproterenol administration, only 14 of the 48 patients $(29 \%)$ in whom the induction endpoint was not reached in the baseline state had tachycardia that could not be reliably provoked. In these 14 patients, the endpoint was not attained because of inability to achieve critical AH lengthening in 6 patients (43\%), fast pathway conduction block in 2 patients (14\%), and slow pathway conduction block in 6 patients $(43 \%$; $\mathrm{P}=\mathrm{NS})$.

Among 13 patients in whom the endpoint was not achieved in the baseline state or during isoproterenol infusion, atropine administration resulted in achievement of the induction endpoint in only one patient. After atropine administration, inability to adequately lengthen the $\mathrm{AH}$ interval, fast pathway block, and slow pathway block were the mechanisms responsible for lack of AVNRT induction in 6 patients $(50 \%), 5$ patients $(42 \%)$, and 1 patient $(8 \% ; \mathrm{P}=\mathrm{NS})$, respectively.

The mechanism responsible for lack of AVNRT induction was the same with each step of the protocol for a particular drug state. However, the mechanism at times differed with the administration of different drugs. For instance, in the 12 patients in whom the tachycardia could never be induced at least seven times, the mechanism responsible was the same in each drug state in 6 patients: the inability to achieve a critical $\mathrm{AH}$ interval in 3 patients, consistent fast pathway block in 1 patient, and consistent slow pathway block in 2 patients 


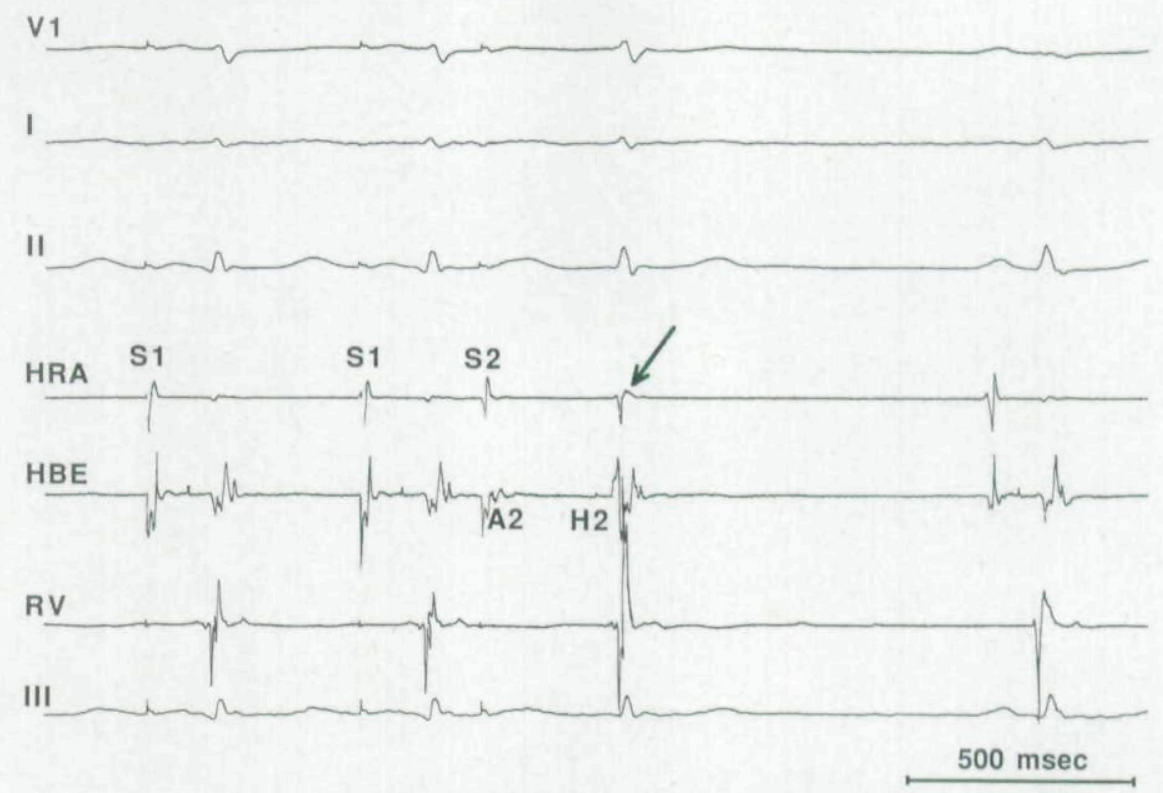

Figure 3. Slow pathway conduction block that limits the induction of AVNRT. Note that $A 2$ is associated with a long A2H2 interval. A subsequent AV nodal echo (arrow) is due to retrograde fast pathway conduction. AVNRT induction is then prevented by anterograde slow pathway block. The tracings and abbreviations are as described in Figure I, except leads VI, I, and II are also shown.

were the mechanisms responsible in each drug state and during each step of the protocol. In the remaining 6 patients, fast pathway block was responsible in the baseline state in each patient, but in only 1 during isoproterenol administration. The isoproterenol had improved fast pathway conduction in the others, but slow pathway conduction block occurred in 2 , and inadequate $\mathrm{AH}$ prolongation prevented reproducible AVNRT induction in 3. After atropine, slow pathway failure $(n=3)$ and lack of adequate $\mathrm{AH}$ prolongation $(\mathrm{n}=3)$ were responsible for the inability to induce AVNRT in at least 7 of 10 attempts.

Four of the 103 patients in this study were diagnosed with AVNRT, but the arrhythmia was never provocable again. The mechanism responsible for no further inductions of AVNRT in these 4 patients was consistent slow pathway conduction failure in 2 patients and consistent inadequate $\mathrm{AH}$ prolongation in 1 patient. In the remaining patient, fast pathway conduction block limited induction in the baseline state, but after isoproterenol and atropine administration, inadequate $\mathrm{AH}$ prolongation prevented tachycardia induction.

There were also eight patients in whom the endpoint was met, but the tachycardia was not induced 10 times. Seven of these 8 patients met the induction endpoint in the baseline state, and one patient achieved the endpoint during isoproterenol infusion. The mechanism responsible for $<10$ in- ductions was lack of critical $\mathrm{AH}$ prolongation in 2 patients and slow pathway conduction block in 5 patients. In the last patient, fast pathway conduction block limited induction in the baseline state and prevented 10 inductions during isoproterenol infusion.

\section{Discussion}

\section{Main Findings}

These results demonstrate that after the diagnosis of AVNRT is established, AVNRT can be in-

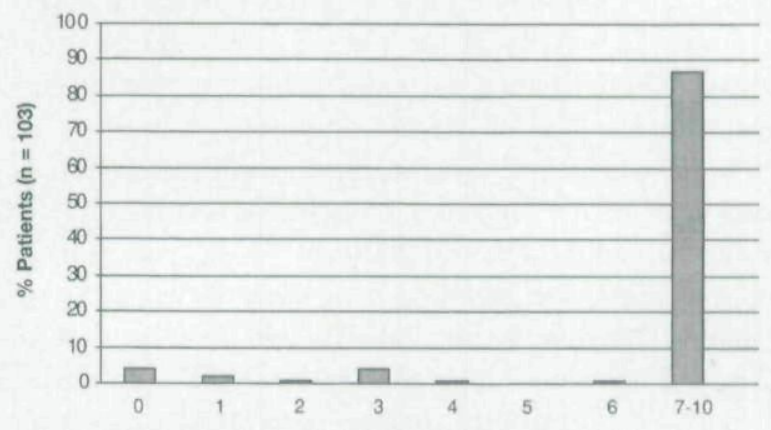

Figure 4. Percentage of patients (y axis) with 0 to 10 successful AVNRT induction attempts, after the diagnosis of typical AVNRT was confirmed. Because the study endpoint was seven or more inductions of AVNRT, patients who met this endpoint were grouped together. 


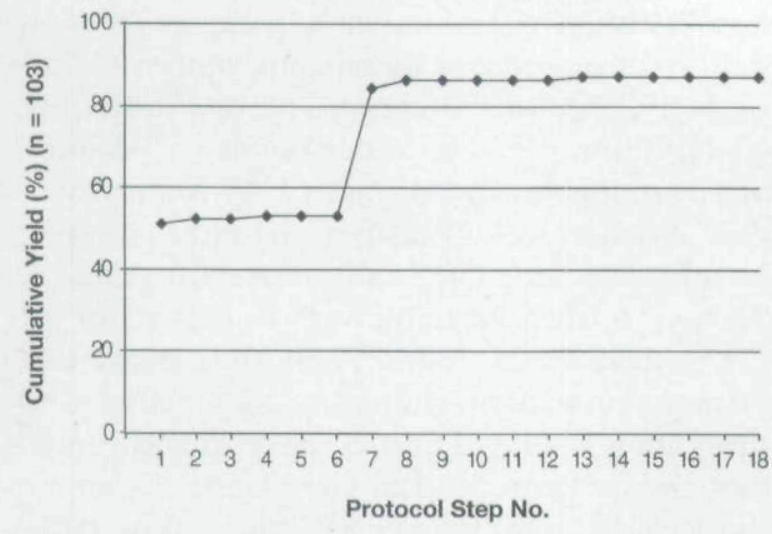

Figure 5. Cumulative yield of AVNRT in seven or more attempts at each step of the pacing protocol. For a complete description of steps 1 through 18, see Table 1 .

duced in at least 7 of 10 attempts in approximately $90 \%$ of patients with proven AVNRT if a variety of pacing and drug interventions are utilized. Fast pathway conduction failure is most commonly responsible for inability to achieve the induction endpoint in the baseline state, but with enhanced adrenergic tone, is rarely the weak link. Approximately $99 \%$ of patients who meet this induction endpoint will do so in the baseline state or after isoproterenol infusion. Atropine administration is rarely helpful when isoproterenol has not been helpful. Likewise, atrial pacing is the effective induction mode in over $90 \%$ of patients.

\section{Autonomic Factors Affecting Noninducibility}

The mechanism responsible for inability to achieve the endpoint of 7 inductions of AVNRT

\section{TABLE 2}

Comparison of Baseline Electrophysiologic Characteristics in Patients in Whom AVNRT was and was not Inducible in $\geq 7$ of 10 Attempts in the Baseline State

\begin{tabular}{lccc}
\hline & \multicolumn{2}{c}{$\geq \mathbf{7}$ Inductions of AVNRT } & \\
\cline { 2 - 3 } & $\begin{array}{c}\text { Yes } \\
(\mathbf{n}=\mathbf{5 5})\end{array}$ & $\begin{array}{c}\text { No } \\
(\mathbf{n}=\mathbf{4 8})\end{array}$ & P Value \\
\hline SCL (msec) & $719 \pm 138$ & $775 \pm 174$ & NS \\
AH (msec) & $82 \pm 20$ & $84 \pm 17$ & NS \\
HV (msec) & $45 \pm 5$ & $39 \pm 6$ & NS \\
FP ERP (msec) & $327 \pm 63$ & $333 \pm 90$ & NS \\
SP ERP (msec) & $263 \pm 42$ & $316 \pm 105$ & $<0.01$ \\
AVBCL (msec) & $345 \pm 56$ & $389 \pm 94$ & $<0.01$ \\
VABCL (msec) & $331 \pm 72$ & $397 \pm 97$ & 0.001 \\
\hline SCL
\end{tabular}

$\overline{\mathrm{SCL}}=$ sinus cycle length; $\mathrm{AH}=$ atrial-His interval; $\mathrm{HV}$ $=$ His-ventricular interval; FP ERP $=\mathrm{AV}$ node fast pathway effective refractory period; SP ERP $=$ AV node slow pathway effective refractory period; $\mathrm{AVBCL}=$ atrioventricular block cycle length; $\mathrm{VABCL}=$ ventricular atrial block cycle length; NS $=$ no statistical difference.
TABLE 3

Electrophysiologic Characteristics in 34 Patients with AVNRT Induced During Isoproterenol Administration

\begin{tabular}{lccc}
\hline & Baseline & Isoproterenol & P Value \\
\hline SCL (ms) & $745 \pm 15$ & $538 \pm 13$ & $<0.0001$ \\
AH (ms) & $83 \pm 18$ & $71 \pm 34$ & $<0.01$ \\
HV (ms) & $45 \pm 4$ & $39 \pm 4$ & NS \\
FP ERP (ms) & $330 \pm 76$ & $233 \pm 34$ & $<0.0001$ \\
SP ERP (ms) & $283 \pm 76$ & $201 \pm 62$ & 0.06 \\
AVBCL (ms) & $365 \pm 79$ & $263 \pm 34$ & $<0.0001$ \\
VABCL (ms) & $365 \pm 92$ & $264 \pm 40$ & $<0.0001$ \\
\hline
\end{tabular}

Abbreviations as in Table 2.

out of 10 attempts in the baseline state was most frequently fast pathway block. This implies that the fast pathway is frequently the weak link for induction in the baseline state, but fast pathway block is rarely responsible for inability to achieve the induction endpoint during autonomic stimulation. This observation suggests that fast pathway conduction is more marginal in the absence of sympathetic stimulation than is slow pathway conduction. Inability to achieve adequate $\mathrm{AH}$ lengthening may be easier to overcome with beta-blocker therapy than with autonomic stimulation. ${ }^{5}$ Additionally, isoproterenol infusion could render the tachycardia more difficult to induce, especially if the fast pathway refractory period were to become shorter than the slow pathway refractory period.

A previous study demonstrated that in the absence of isoproterenol, the ventricular response during atrial fibrillation slows after slow pathway ablation for AVNRT. ${ }^{6}$ However, recent observations demonstrate that the ventricular response increases when adrenergic tone is enhanced with isoproterenol. ${ }^{7}$ This phenomenom may also be due to the responsiveness of the fast pathway to adrenergic stimulation.

\begin{tabular}{|c|c|c|c|c|c|}
\hline \multicolumn{6}{|c|}{$\begin{array}{c}\text { TABLE 4 } \\
\text { Mechanisms Responsible for Nonreproducible } \\
\text { Induction of AVNRT }\end{array}$} \\
\hline & & \multicolumn{3}{|c|}{ Mechanism } & \multirow[b]{2}{*}{ P Value } \\
\hline & $\mathbf{n}$ & No $\uparrow \mathbf{A H}$ & $\begin{array}{c}\text { FP } \\
\text { Block }\end{array}$ & $\begin{array}{c}\text { SP } \\
\text { Block }\end{array}$ & \\
\hline Baseline & 49 & $10(20 \%)$ & $24(49 \%)$ & $15(31 \%)$ & 0.02 \\
\hline Isoproterenol & 14 & $6(43 \%)$ & $2(14 \%)$ & $6(43 \%)$ & NS \\
\hline Atropine & 12 & $6(50 \%)$ & $5(42 \%)$ & $1(8 \%)$ & NS \\
\hline
\end{tabular}




\section{Previous Studies}

In the present study, after isoproterenol administration, the induction endpoint was still not achieved in approximately one third of patients, despite significantly improving anterograde and retrograde conduction. Isoproterenol has previously been shown to be beneficial for the induction of AVNRT in patients with dual AV nodal physiology, with and without sustained reentry. ${ }^{1}$ However, a balance of anterograde slow pathway and retrograde fast pathway conduction is required for tachycardia induction. ${ }^{4}$

Atropine was rarely helpful in inducing sustained AVNRT for patients in whom isoproterenol administration did not cause reproducible AVNRT induction. Atropine consistently improved AV and VA conduction, yet this was not adequate to reproducibly induce AVNRT. The results of a previous study suggested that atropine can be helpful at inducing AVNRT. ${ }^{2}$ Because atropine improves anterograde and retrograde conduction, it is not surprising that atropine may aid in the induction of AVNRT. ${ }^{2}$ In the present study, isoproterenol administration and atropine administration had similar effects on AV and VA conduction. Perhaps this is why atropine was rarely helpful in achieving the induction endpoint after isoproterenol had been ineffective.

\section{Limitations}

The design of this study required that AVNRT be inducible at least once for the patient to be included in the study. There may be some patients with AVNRT who are completely noninducible, and so these data may underestimate the true frequency of nonreproducible induction of AVNRT. An additional limitation of this study is that only a single dose of isoproterenol and atropine were used. The results of this study may have been different if other doses were used.

\section{Clinical Implications}

Some patients with documented PSVT are completely noninducible, suggesting that their tachycardia may be automatic atrial tachycardia. However, the results of the present study suggest that the diagnosis of AVNRT is still possible and should be entertained when dual AV nodal physiology, with or without echoes, is present. In this instance, if prior medical therapy has been ineffective, empiric slow pathway ablation may be a reasonable therapeutic option. Additionally, the lack of reproducibly inducible AVNRT may lead to recurrences of AVNRT after an apparently successful ablation procedure, because lack of tachycardia induction may be mistaken for a successful procedure. Finally, AVNRT is often inducible with isoproterenol but not in the baseline state. These data imply that isoproterenol administration in conjunction with programmed stimulation of the atria and ventricles should be utilized to completely document the lack of inducible AVNRT after slow pathway ablation. ${ }^{8}$

Acknowledgment: The authors wish to thank Allyson Navyac for her excellent secretarial support.

\section{References}

1. Huycke EC, Lai WT, Nguyen NX, et al: Role of intravenous isoproterenol in the electrophysiologic induction of atrioventricular node reentrant tachycardia in patients with dual atrioventricular node pathways. Am J Cardiol 1989;64:1131-1137.

2. Wu D, Denes P, Bauernfeind R, et al: Effects of atropine on induction and maintenance of atrioventricular nodal reentrant tachycardia. Circulation 1979;59: 779-788.

3. Josephson ME: Clinical Cardiac Electrophysiology: Techniques and Interpretations. Lea \& Febiger, Philadelphia, 1993, pp. 181-224.

4. Denes P, Wu D, Amat-y-Leon F, et al: The determinants of atrioventricular nodal re-entrance with premature atrial stimulation in patients with dual A-V nodal pathways. Circulation 1977;56:253-259.

5. Kay GN, Epstein AE, Dailey SM, et al: Selective radiofrequency ablation of the slow pathway for the treatment of atrioventricular nodal reentrant tachycardia: Evidence for involvement of perinodal myocardium within the reentrant circuit. Circulation 1992;85:1675-1688.

6. Blanck Z, Dhala AA, Sra J, et al: Characterization of atrioventricular nodal behavior and ventricular response during atrial fibrillation before and after a selective slow-pathway ablation. Circulation 1995;91:10861094.

7. Strickberger SA, Weiss R, Goyal R, et al: The effect of slow pathway ablation on the ventricular rate in atrial fibrillation during sympathetic stimulation and autonomic blockade. Circulation 1995;92:I-84.

8. Hummel JD, Strickberger SA, Williamson BD, et al: Effect of residual slow pathway function on the time course of recurrences of atrioventricular nodal reentrant tachycardia after radiofrequency ablation of the slow pathway. Am J Cardiol 1995;75:628-630. 
This document is a scanned copy of a printed document. No warranty is given about the accuracy of the copy. Users should refer to the original published version of the material. 